\title{
IRI-MAC: An Improved Receiver Initiated MAC Protocol for Wireless Sensor Network
}

\author{
Md. Abir Hossain \\ Department of Information and \\ Communication Technology \\ (ICT), \\ Mawlana Bhashani \\ Science and Technology \\ University (MBSTU), \\ Bangladesh
}

\author{
Md. Mahfuz Reza \\ Department of Computer \\ Science and \\ Engineering (CSE), \\ Mawlana Bhashani Science \\ and Technology University \\ (MBSTU), Bangladesh
}

\author{
Nafiul Bari Khan \\ Department of Computer \\ Science and Engineering \\ (CSE), Mawlana Bhashani \\ Science and Technology \\ University (MBSTU), \\ Bangladesh
}

\author{
Md. Tasnim Alam \\ Department of \\ Information and Communication \\ Engineering, Islamic University, \\ Bangladesh
}

\begin{abstract}
Now a day's wireless sensor network takes much attention to researcher. A lot of Medium Access Control (MAC) protocol has been proposed to improve the efficiency in case of increase throughput and energy preservation. ReceiverInitiated MAC(RI-MAC) is one of the most popular MAC protocol due to its ultra-low duty cycle and good throughput. But RI-MAC major disadvantages are exposed terminal collision problem and hidden terminal collision problem. In this paper we only addressed exposed terminal problem using an unique time Clear Channel Assessment(CCA).According to Improved Receiver-Initiated MAC(IRI-MAC) protocol the receiver beacon is not respond immediately but wait a random time and perform a CCA either the channel is clear. If channel clear then transmit its data immediately. More precisely, we provide such an equation that generate an unique CCA time for each node in order to avoid collision for exposed terminal problem. Our proposed system is implemented in omnet/Mixim platform and result shows that IRI-MAC perform better than that of RC-MAC and RI-MAC.
\end{abstract}

\section{Keywords}

Medium Access protocol, Asynchronous duty cycling, wireless sensor network.

\section{INTRODUCTION}

In Wireless Sensor Networks (WSN), a number of tiny wireless sensor nodes are used to exchange data for a long period of time with a non-rechargeable limited battery power capacity. A major power consuming component of a sensor node is the radio, which is controlled by the MAC protocol[17]. Therefore, energy consumption is one of the most significant issues which is taking concerns when design a protocols or application for sensors. In WSN, overhearing, idle listening and collisions are the most familiar causes of energy consumption [1].

Overhearing is a phenomenon of wireless communication where a wireless node listens to a packet transmitted by its adjacent nodes all the time. In idle listening, a sensor node turns on its radio and listening for a possible packet to be received even though nothing has been sent. On the other hand, collision occurs when many nodes are transmitting data packet in the channel at the same time. Using medium access protocol such as CSMA/CA (Carrier sense multiple access with collision avoidance) collisions can be minimized [2] that ensures the exchange of control message to hold back the wireless channel before each data transmission [2].

A lot of ideas have been proposed to solve the idle listening problem make use of duty cycling technique [3, 4,]. In duty cycling technique, every sensor node turns radio on only periodically, alternating between active and sleeping states such as EE-RI-MAC[18]. In active state, nodes are able to transmit or receive data message on the other hand in sleeping state, the node totally turns off its radio to save energy and avoid all data transmission and reception.

In different MAC protocols[6-8,10,11] imply different mechanism to transmit data at different time. Their transmission is offsetting by different influential factor that could cause avoid collision and data is transmitted to target receiver easily.

\section{BACKGROUND}

MAC protocols usually support routing mechanism and apply different rules and disciplines that guide the sensor nodes in order to collision free data transmission. Contention based duty cycle MAC protocols can be classified into two different categories: synchronous and asynchronous. In Synchronous MAC protocols sensor nodes synchronize each other schedule. Some node follows other node's schedule some nodes broadcast their own schedule. In Synchronous MAC, such as T-MAC [5], S-MAC [4], DW-MAC [6] and RMAC [7], the protocol divides the duty cycle in two section as active state and sleep state. In active state, it is decided which node transmit in the upcoming sleep state and in sleep state only the scheduled node transmit their data packet without any collision. In Essence in sleep state the all non-schedule node goes to sleep. This approach significantly decreases idle listening time, but the essential synchronization initiates extra overhead and complexity, and a node may require to wake up several times if its neighbors are on different schedules. Conversely, asynchronous duty-cycling MAC protocols do not require such synchronization rather than synchronous MAC protocols. That's why asynchronous MAC protocols are most popular due to their low duty cycle and low 
communication overhead. Most asynchronous MAC protocols includes X-MAC [8], B-MAC [9] and WiseMAC [10]) or receiver-initiated RI-MAC [11, 12]. With the sender initiated approach, a sender transmits a preamble before a packet transmission to notify the receiver of the upcoming packet. WiseMAC pioneered predictive wakeup in sensor network MAC protocols by fixing the node wakeup interval, thereby enabling a sender to deduce future receiver wakeup times and send a shortened wakeup preamble shortly before the receiver wakes up. However, this fixed node wakeup interval may allow repeated node wakeup schedule collisions, particularly in dense networks, thus degrading performance. With the receiver-initiated approach, in contrast, sender preambles are replaced with receiver wakeup beacons; as the beacon is substantially shorter than a preamble; wireless bandwidth usage and collisions are reduced [12]. Some other energy efficient routing protocols have been proposed [13-16].

A lot of modification perform on RI-MAC[11].RC-MAC[19] avoids data collision by adding another CCA before data transmission. Another EE-RI-MAC[18] saves energy consumption of RI-MAC. As well as SA-RI-MAC[20] provide a unique mechanism to reduce number of collision but still collision occurs due to simultaneous data transmission.

We propose an unique CCA time based IRI-MAC protocol That capable to determine the non-repeating CCA-time due to avoid collision among nodes. The rest of the paper organizes as Section .3 provides the IRI-MAC protocol details section. 4 provides the detail simulation results as well as section.5 represent the conclusion of the paper and section. 6 provides the reference.

\section{IRI-MAC DESIGN OVERVIEW}

\subsection{Exposed terminal and Hidden terminal collision problem}

Two or more wireless sensor nodes that are in the same sensing region and collide each other due to simultaneous data transmission is called exposed terminal collision problem. Almost all sensor node has transmission range and interference range. The different MAC protocol avoid this collision by offsetting their transmission time.

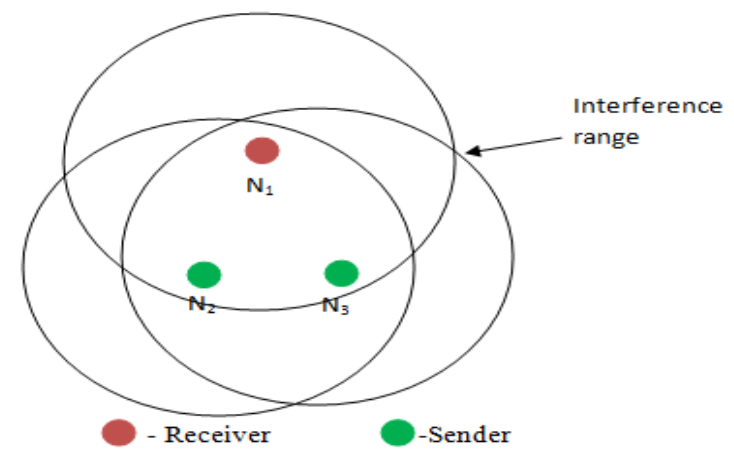

Fig.1: Exposed Terminal problem

Here node $\mathrm{N}_{2}$ and $\mathrm{N}_{3}$ are in same sensing region. In order to avoid exposed terminal problem, we have to offsett their transmission by any factor.

The Hidden node is such a node that is out of the interference range of other node. Due to hidden node, if collision is occur, that is called hidden terminal problem.

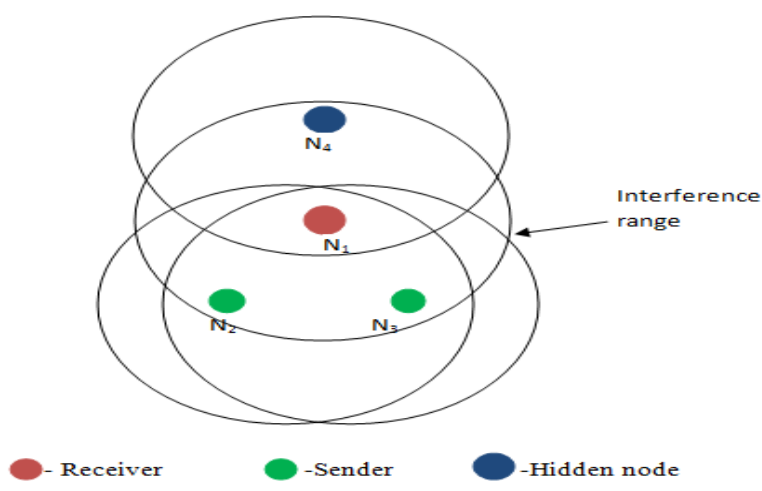

Fig.2: hidden Terminal problem.

Here Node $\mathrm{N}_{4}$ is out of the interference range of node $\mathrm{N}_{2}$ and $\mathrm{N}_{3}$. Thus any data transmission of sender $\mathrm{N}_{2}$ and $\mathrm{N}_{3}$ to Receiver $\mathrm{N}_{1}$ may collide due to $\mathrm{N}_{4}$ node packet. According to this diagram $\mathrm{N}_{4}$ act as hidden node for node $\mathrm{N}_{2}$ and $\mathrm{N}_{3}$.

\subsection{Collision problem in Existing MAC protocols}

Due to exposed and Hidden terminal node a lot of collision occur in Receiver-Initiated MAC(RI-MAC) and ReceiverCentric MAC(RC-MAC). In RI-MAC, the sender awake and wait random time for receiver beacon packet. The target receiver also awake randomly and transmit its beacon packet announcing that it is ready to receive packet. The intended node those are waiting to transmit data regarding this receiver node immediately transmit after receiving the receiver beacon packet.

The collision occur when two or multiple nodes listen the beacon at the same time and immediately transmit their data to the target receiver.

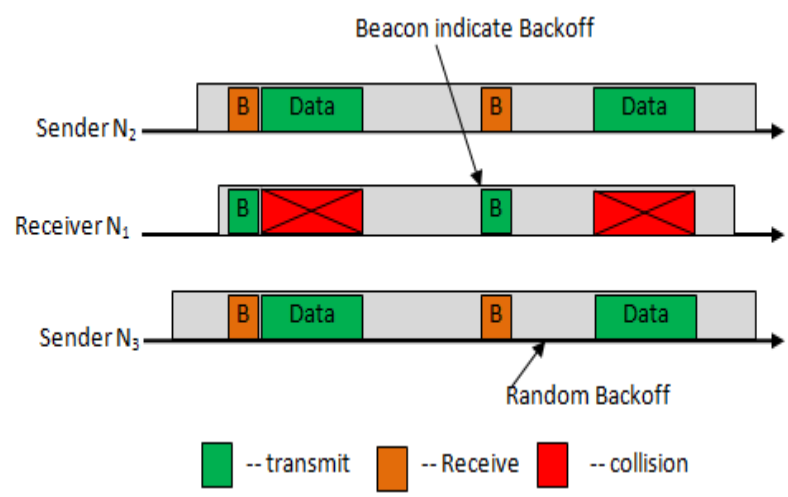

Fig. 3: Collision problem in RI-MAC.

Figure 3 shows the timing diagram of different sender and receiver node. Node $\mathrm{N}_{2}$ and $\mathrm{N}_{3}$ both wish to transmit data to receiver node $\mathrm{N}_{1}$. But the sender node $\mathrm{N}_{2}$ and $\mathrm{N}_{3}$ listen the receiver beacon at the same time and immediately transmit their data to the target receiver that results a collision at the receiving end.

After colliding packet of nodes, the receiver transmit a beacon. The beacon packet serve two purposes, one: it mentioning that the transmitted packet is not successfully received and second: it holds a a backoff value to indicate that transmit again after taking a this backoff. If nodes transmit again simultaneously after taking random backoff that would lead again a collision. According to RI-MAC, after 
several trial if any node is failed for a successful transmission then take a binary exponential backoff.

The backoff mechanism in RI-MAC shows poor performance in case of throughput and consume much energy for lot of packet retransmission. This problem is largely mitigated by RC-MAC. According to RC-MAC, if any sender receive beacon packet from target receiver node, then avoid their immediate data transmission in order to avoid data collision with neighboring nodes. The sender waits for a random time and then perform a Clear Channel Assessment (CCA) to confirm no ongoing transmission in the channel. After performing a successful CCA, if it get the channel clear then immediately transmit its data to target receiver.

But collision occurs if two nodes perform CCA at the same time then gets the channel clear and if transmit data immediately that could cause a collision.
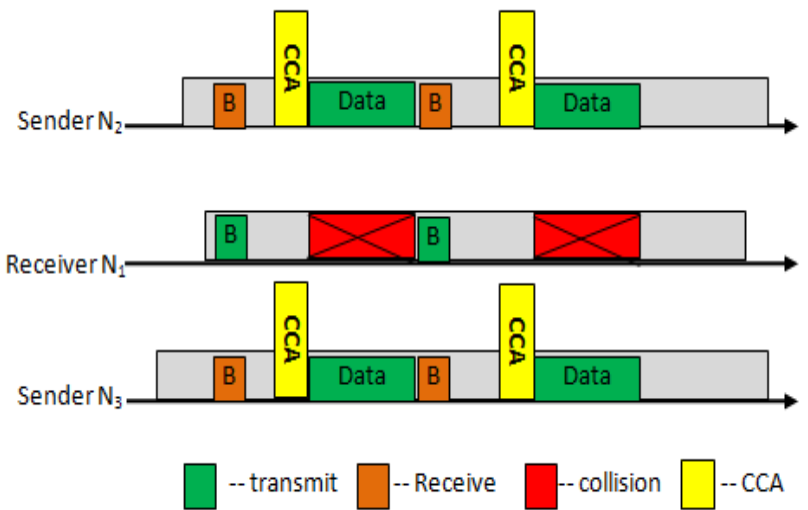

Fig. 4: Collision problem in RC-MAC.

In Figure 4 , if sender node $\mathrm{N}_{2}$ and $\mathrm{N}_{3}$ perform CCA at the same time then gets the channel clear. If two node gets the channel clear then transmit immediately that lead a collision at target receiving end. So there is a challenge to detect a unique time to perform CCA and transmit data.

\subsection{Probability of calculating the different random number}

Let for a range $\left[\mathrm{T}_{1}, \mathrm{~T}_{2}\right]$ there are $\mathrm{k}$ choice for $\mathrm{n}$ nodes. Then

first node choose a random number .

The second node choose another random number with probability $\frac{1}{k} \quad$ and the second node's probability of having not the same random number chosen by other node is $1-\frac{1}{k}$ $=\frac{k-1}{k}$

the third node's probability of choosing a random number is $\frac{2}{k}$ and third node's probability of having not the same random number chosen by other node is $1-\frac{2}{k}=\frac{k-2}{k}$

For $\mathrm{n}^{\text {th }}$ node the probability of choosing a random number is $\frac{n-1}{k}$ and $\mathrm{n}^{\text {th }}$ node's probability of having not the same random number chosen by other node is $1-\frac{n-1}{k}=\frac{k-n+1}{k}$

so, the total probability of choosing a random number that are not chosen by other node is

$$
\rho=1-\frac{\mathrm{k}(\mathrm{k}-1)(\mathrm{k}-2) \ldots(\mathrm{k}-\mathrm{n}+2)(\mathrm{k}-\mathrm{n}+1)}{\mathrm{k}^{\mathrm{n}}}
$$

$$
\rho=1-\frac{K !}{k^{n}(k-n) !}
$$

\subsection{Unique CCA time Calculation}

We use Linear Congruent Generator(LCG) to generate unique CCA time in order to avoid collision among neighboring nodes. The traditional LCG equation[21] is

$X_{n}=\left(a X_{n-1}+C\right) \bmod m$

where $\mathrm{a}, \mathrm{c}$ and $\mathrm{m}$ are prime number and $\mathrm{c}$ and $\mathrm{m}$ are relative prime value. $X_{n-1}$ is previous state random number and $\mathrm{Xn}$ is current state random value.

we modified the LCG equation in our paper and determine unique CCA time as

\section{CCA Time $=$}

(node Id $*$ random prime +

relative prime) mod (total node number $* 2$ ).

According to equation (3) the node id is the sensor nodes self Id, random prime is a random prime value from $2 \sim 2 *$ total node number range and relative prime is the nearest prime value of the $2 *$ total node number.

\subsection{Proposed IRI-MAC diagram}

The improved RI-MAC focus on the unique CCA time. According to IRI-MAC each node sense the medium at nonrepeating unique CCA time in order to avoid data collision. If any node gets the channel busy then wait for a random time until it gets any beacon from the target receiver node. For each beacon transmission the receiver node broadcast a unique prime value within the range [2 2*total node number] and each node generate a unique CCA time using equation (3) and receiver broadcast prime value.

Though each node can capable to generate unique CCA time thus probability of collision can be greatly minimized and each node accurately detect either channel is clear or busy. The decision making of attempting data transmission can be more exact though CCA result is accurate.
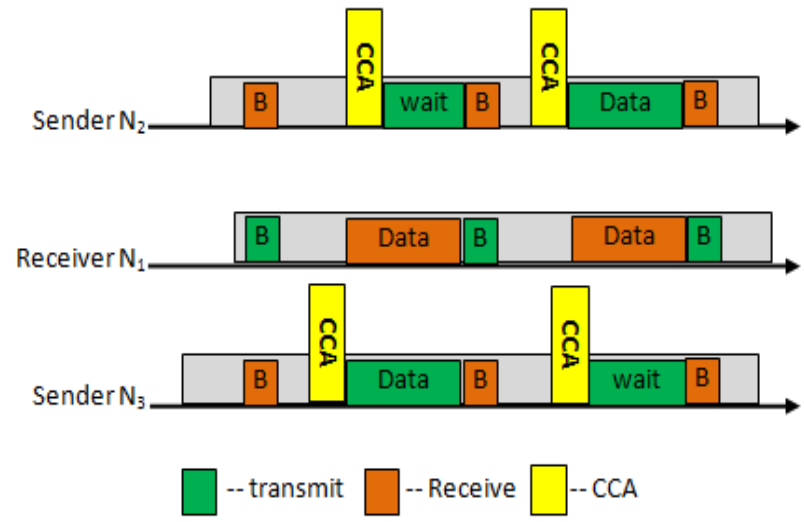

Fig. 5: proposed IRI-MAC

The sensor nodes are randomly distributed and sender $\mathrm{N}_{2}$ and $\mathrm{N}_{3}$ wants to transmit data to target receiver $\mathrm{N}_{1}$. The receiver transmit a beacon to inform the neighboring node that it is ready to receive data. After having the beacon packet with random prime each node $\mathrm{N}_{2}$ and $\mathrm{N}_{3}$ generate their CCA time and get decision based on the result of the CCA. For the first time sender $\mathrm{N}_{3}$ gets the channel clear and transmit its data and sender $\mathrm{N}_{2}$ waits until it gets another beacon from the target receiver and for the second time sender $\mathrm{N}_{2}$ gets the channel clear and transmit its packet. 


\subsection{Beacon Format}

\begin{tabular}{|l|l|l|l|l|l|l|l|}
\hline $\begin{array}{c}\text { Hardware } \\
\text { preamble }\end{array}$ & $\begin{array}{c}\text { Frame } \\
\text { length }\end{array}$ & FCF & SRC & BW & DST & $\begin{array}{c}\text { Random } \\
\text { Prime }\end{array}$ & FCS \\
\hline
\end{tabular}

Fig. 6: Beacon format for IRI-MAC receiver

we slightly modify the beacon format for our proposed IRIMAC. we add a new field "random prime" in order to generate unique CCA time. Based on the Random Prime value each node calculate its own CCA time.

\section{SIMULATION RESULTS}

we simulate our proposed IRI-MAC protocol in Omnet/Mixim platform. The sensor nodes are randomly distributed among a $100 \times 100 \mathrm{~m}$ area. The sensor nodes transmission range is $250 \mathrm{~m}$ and carrier sensing range is $500 \mathrm{~m}$. The Bandwidth is $512 \mathrm{kbps}$ and CCA check delay is random. we simulate the program and run it 50 times with 100s and the result place in average number of collision of packets and average throughput in kbps. The simulations results of average throughput is given below:

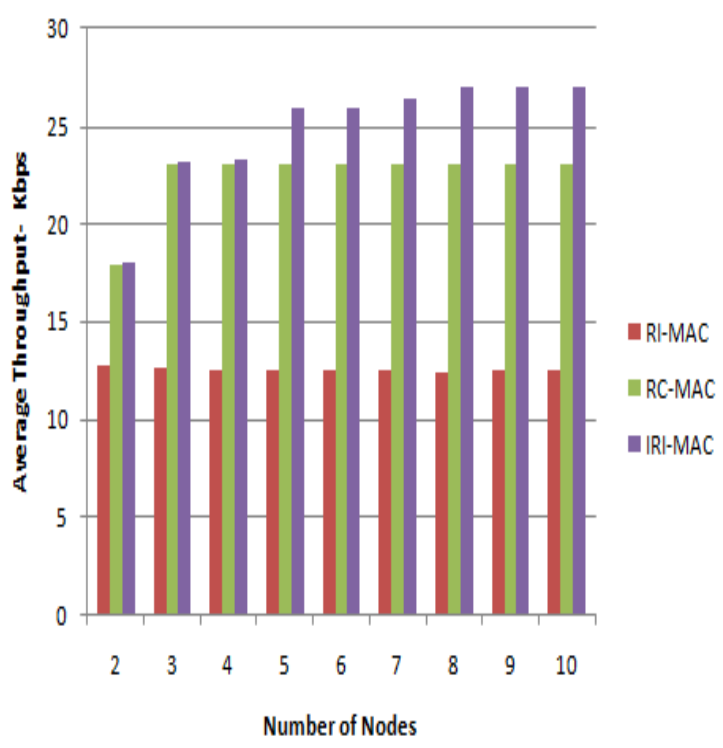

Fig. 7: Average Throughput comparison.

According to graph result shown in figure 7, the RI-MAC throughput is very low and it improve in RC-MAC by applying CCA before transmitting any data packet in order to avoid collision. The result is more improved in IRI-MAC by applying the unique CCA time in order to detect the unique time to CCA and get the accurate decision based on the condition. Another important observation is that when the number of node increase the result in RI-MAC is remain constant but in case of RC-MAC and IRI-MAC the value is changing due to collision problem among different nodes. The collision happened due to exposed terminal problem of same time CCA check and also for hidden node collision problem.

The average number of packet collision is also observed in our analysis. The analysis results are shown in figure 8 .

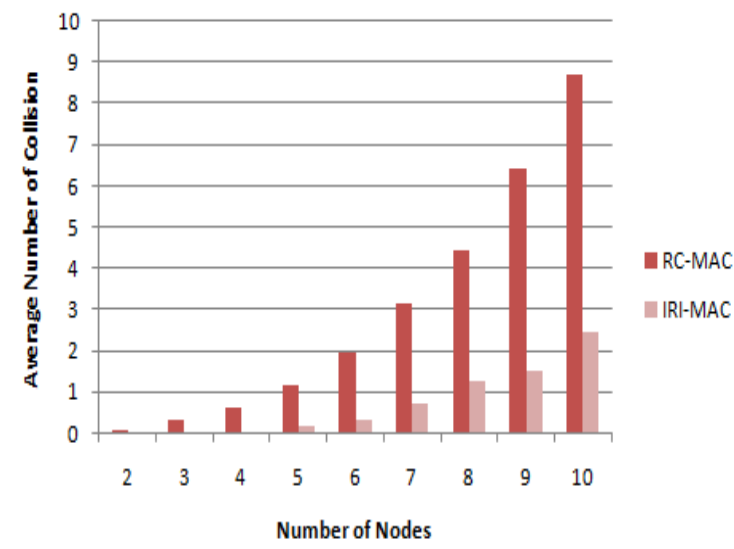

Fig.8: Average collision comparison

According to graph results shown in figure 8, when node number is relatively low, the value of average collision is also significantly low. But when the number of nodes increases in the network, the number of collision is proportionally increases. In RC-MAC the CCA check perform for a range with random value that cause a lot of collision in increase of number of nodes. This is because any two nodes can generate the same value twice that would lead a collision and require retransmission. RC-MAC use NAV to avoid collision for Hidden node terminal problem but it arise mask node problem that could cause collision at other end.

In IRI-MAC, we perform accurate non-repeated CCA time detection that greatly minimize the collision but still collision occur due to hidden node problem.

\section{CONCLUSION}

we propose an IRI-MAC in which each node can capable to determine a unique time of CCA that avoids collision among nodes. If node gets the channel clear then immediately transmit their data packet. During an ongoing transmission if another node perform a CCA and if the sensor node is in the same interference range then it could detect the channel is busy and avoid data transmission. The nodes waits for a random time to receiver another beacon from target receiver node. After having a new beacon it again compete to win the channel. We use different range of nodes to calibrate our protocol and in all cases our result provide better results compare to RI-MAC and RC-MAC. We try to avoid hidden terminal collision in our future work.

\section{REFERENCES}

[1] Kredo, K., \& Mohapatra, P. (2007). Medium access control in wireless sensor networks. Computer networks, 51(4), 961-994.

[2] Kleinrock, L., \& Tobagi, F. A. (1975). Packet switching in radio channels: Part I--carrier sense multiple-access modes and their throughput-delay characteristics. Communications, IEEE Transactions on, 23(12), 14001416.

[3] Polastre, J., Hill, J., \& Culler, D. (2004, November). Versatile low power media access for wireless sensor networks. In Proceedings of the 2nd international conference on Embedded networked sensor systems (pp. 95-107). ACM.

[4] Ye, W., Heidemann, J., \& Estrin, D. (2002). An energyefficient MAC protocol for wireless sensor networks. In INFOCOM 2002. Twenty-First Annual Joint Conference 
of the IEEE Computer and Communications Societies. Proceedings. IEEE (Vol. 3, pp. 1567-1576). IEEE.

[5] Van Dam, T., \& Langendoen, K. (2003, November). An adaptive energy-efficient MAC protocol for wireless sensor networks. In Proceedings of the 1st international conference on Embedded networked sensor systems (pp. 171-180). ACM.

[6] Sun, Y., Du, S., Gurewitz, O., \& Johnson, D. B. (2008, May). DW-MAC: a low latency, energy efficient demand-wakeup MAC protocol for wireless sensor networks. In Proceedings of the 9th ACM international symposium on Mobile ad hoc networking and computing (pp. 53-62). ACM.

[7] Shu Du, Amit Kumar Saha, and David B. Johnson. RMAC: A Routing-Enhanced Duty-Cycle MAC Protocol for Wireless Sensor Networks. In Proceedings of the $26^{\text {th }}$ Annual IEEE Conference on Computer Communications (INFOCOM 2007), pages 1478-1486, May 2007.

[8] Buettner, M., Yee, G. V., Anderson, E., \& Han, R. (2006, October). X-MAC: a short preamble MAC protocol for duty-cycled wireless sensor networks. In Proceedings of the 4th international conference on Embedded networked sensor systems (pp. 307-320). ACM.

[9] Polastre, J., Hill, J., \& Culler, D. (2004, November). Versatile low power media access for wireless sensor networks. In Proceedings of the 2nd international conference on Embedded networked sensor systems (pp. 95-107). ACM.

[10] El-Hoiydi, A., \& Decotignie, J. D. (2004). WiseMAC: An ultra low power MAC protocol for multi-hop wireless sensor networks. In Algorithmic Aspects of Wireless Sensor Networks (pp. 18-31). Springer Berlin Heidelberg..

[11] Sun, Y., Gurewitz, O., \& Johnson, D. B. (2008, November). RI-MAC: a receiver-initiated asynchronous duty cycle MAC protocol for dynamic traffic loads in wireless sensor networks. In Proceedings of the 6th ACM conference on Embedded network sensor systems (pp. 114). ACM

[12] Estrin, D., Govindan, R., Heidemann, J., \& Kumar, S. (1999, August). Next century challenges: Scalable coordination in sensor networks. In Proceedings of the 5th annual ACM/IEEE international conference on Mobile computing and networking (pp. 263-270). ACM.
[13] Schurgers, C., \& Srivastava, M. B. (2001). Energy efficient routing in wireless sensor networks. In Military communications conference, 2001. MILCOM 2001. Communications for network-centric operations: Creating the information force. IEEE (Vol. 1, pp. 357361). IEEE

[14] Rana, S., Bahar, A. N., Islam, N., \& Islam, J. (2015). Fuzzy based Energy efficient multiple cluster head selection routing protocol for wireless sensor networks. International Journal of Computer Network and Information Security (IJCNIS), 7(4), 54.

[15] Yu, C., Lee, B., \& Yong Youn, H. (2003). Energy efficient routing protocols for mobile ad hoc networks. Wireless communications and mobile computing, 3(8), 959-973.

[16] Islam, M. M., Uddin, M. A., Bahar, A. N., Islam, A. K., \& Al Mamun, M. S. (2014). Energy Efficient Routing Mechanism for Mobile Ad Hoc Networks. International Journal of Computer Science Engineering (IJCSE), 3(1), 9-20

[17] Huang, P., Xiao, L., Soltani, S., Mutka, M. W., \& Xi, N. (2013). The evolution of MAC protocols in wireless sensor networks: A survey. Communications Surveys \& Tutorials, IEEE, 15(1), 101-120.

[18] Yong, Y. T., Chow, C. O., Kanesan, J., \& Ishii, H. (2011). EE-RI-MAC: An energy-efficient receiverinitiated asynchronous duty cycle MAC protocol for dynamic traffic loads in wireless sensor networks. International Journal of Physical Sciences, 6(11), 26332643.

[19] Huang, P., Wang, C., Xiao, L., \& Chen, H. (2010, June). RC-MAC: A receiver-centric medium access control protocol for wireless sensor networks. In Quality of Service (IWQoS), 2010 18th International Workshop on (pp. 1-9). IEEE.

[20] Henna, S. (2011). Sa-ri-mac: sender-assisted receiverinitiated asynchronous duty cycle mac protocol for dynamic traffic loads in wireless sensor networks. In Mobile Lightweight Wireless Systems (pp. 120-135). Springer Berlin Heidelberg.

[21] Linear Congruential Random Number Generator, princeton university education; [access on 5.17.2016], available:http://www.cs.princeton.edu/courses/archive/sp ring03/cs126/assignments/cycle.html 